\title{
Tratamiento con clavo endomedular de las fracturas metafisarias proximales y distales de tibia. Abordaje pararrotuliano medial en posición de semiextensión
}

\author{
Sebastián Pereira, Gabriel Vindver, Fernando Bidolegui \\ Servicio de Ortopedia y Traumatología, Hospital Sirio Libanés, ECICARO, Ciudad Autónoma de Buenos Aires, Argentina
}

\section{RESUMEN}

Objetivo: El enclavado endomedular de las fracturas metafisarias de tibia se asocia con algunas complicaciones relacionadas con la necesidad de flexionar la rodilla durante la introducción del clavo endomedular con la técnica infrarrotuliana clásica. Es por ello, que se han diseñado diferentes abordajes para la colocación del clavo en una posición de semiextensión de rodilla. El objetivo fue evaluar nuestros resultados, de forma retrospectiva, con el abordaje pararrotuliano medial en semiextensión, para el tratamiento de las fracturas metafisarias proximales y distales de tibia con clavo endomedular. Materiales y Métodos: Se incluyó a 23 pacientes con un seguimiento posoperatorio mínimo de un año. Doce eran fracturas distales de tibia; 9 , proximales y 2 , segmentarias. Se evaluaron el rango de movilidad de la rodilla, el dolor posoperatorio con la escala de Lysholm, el eje posoperatorio y la tasa de consolidación. Resultados: El arco de movilidad de la rodilla fue de $125^{\circ}$ (rango 110-140). Al año de la cirugía, 16 de 23 pacientes no tenían dolor, 5 de 23 refirieron un leve dolor durante la actividad física y 2, dolores intensos durante la actividad física. En todos los casos, se consiguió un eje posoperatorio aceptable y la consolidación ósea. Un paciente requirió un aumento con una placa e injerto óseo. Conclusión: La colocación de un clavo de tibia a través de un abordaje pararrotuliano medial con la rodilla en semiextensión es una técnica segura y simple para el tratamiento de las fracturas metafisarias de tibia.

Palabras clave: Fracturas metafisarias; tibia; clavo endomedular; semiextensión.

Nivel de Evidencia: IV

Intramedullary nailing treatment of proximal and distal tibia metaphyseal fractures.

Medial parapatellar approach in semi-extended position

\begin{abstract}
Objective: To review the results of a series of metaphyseal tibial fractures treated with intramedullary nailing (IMN) in a semiextended position using a medial parapatellar approach. To report reduction quality, bone consolidation, range of motion (ROM) and postoperative knee pain at final follow-up. Materials and Methods: Twenty-three metaphyseal tibia fractures were treated with IMN. Twelve were distal tibial fractures (AO/OTA 43A), nine were proximal tibial fractures (AO/OTA 41A2/3), and two had segmental tibia fractures (AO/OTA 42C2). The minimum follow-up was 1 year. Results: Radiograph angulation at the fracture site was $<5$ degrees. All patients achieved bone healing. One patient (AO/OTA 42C2) required plate augmentation and bone autografting. Twenty-one out of 23 patients had knee pain scores of $>20$ according to the Lysholm scale. Conclusion: The parapatellar approach in a semi-extended position is a safe and useful technique for IMN of metaphyseal tibia fracture with no associated increased postoperative knee pain.
\end{abstract}

Key words: Metaphyseal tibial fracture; intramedullary nail; semi-extended position.

Level of Evidence: IV

Recibido el 24-3-2019. Aceptado luego de la evaluación el 26-8-2019 • Dr. SEBASTIÁN PEREIRA • sebopereira@ hotmail.com (ID

Cómo citar este artículo: Pereira S, Vindver G, Bidolegui F. Tratamiento con clavo endomedular de las fracturas metafisarias proximales y distales de tibia. Abordaje pararrotuliano medial en posición de semiextensión. Rev Asoc Argent Ortop Traumatol 2020;85(2):99-106. https://doi.org/10.15417/issn.1852-7434.2020.85.2.974 


\section{INTRODUCCIÓN}

La fijación interna con un clavo endomedular es el método de elección para el tratamiento de las fracturas diafisarias de tibia. En las fracturas metafisarias de tibia tanto proximal como distal, el método de fijación es más controvertido. Los clavos endomedulares, incluso los fresados de mayor diámetro, no consiguen obtener contacto cortical en el amplio canal metafisario. Sumado a esto, se ha señalado que la escasa fijación conseguida con los tornillos de bloqueo de los clavos diseñados para fracturas diafisarias es responsable de la alta tasa de deseje posoperatorio cuando se los compara con las placas.${ }^{1-4}$ Sin embargo, el desarrollo de nuevas técnicas quirúrgicas y las mejoras en el diseño de clavos con bloqueos multidireccionales han incrementado el interés por el uso de clavos para la fijación de las fracturas metafisarias de tibia. ${ }^{5}$

La introducción del clavo de tibia a través de un abordaje infrarrotuliano hace difícil conseguir y mantener la reducción de la fractura debido a la necesidad de flexionar la rodilla para introducir el clavo. Con el objetivo de evitar estas dificultades, se han diseñado diferentes abordajes para introducir el clavo en una posición de semiextensión. ${ }^{6-8}$

El objetivo de este estudio fue evaluar nuestros resultados con la técnica del enclavado endomedular de tibia a través de un abordaje pararrotuliano interno en semiextensión para el tratamiento de las fracturas metafisarias proximales y distales de tibia.

\section{MATERIALES Y MÉTODOS}

Entre enero de 2016 y diciembre de 2017, 57 pacientes con fractura de tibia fueron operados con un clavo endomedular. Los criterios de inclusión fueron: fracturas de tibia con compromiso del extremo proximal o distal, sin extensión articular, abordaje quirúrgico pararrotuliano y seguimiento posoperatorio $\geq 1$ año. Los criterios de exclusión fueron: fracturas diafisarias, fracturas patológicas, esqueleto inmaduro, seguimiento posoperatorio $<1$ año y abordaje quirúrgico transtendón rotuliano. Una vez aplicados los criterios de inclusión y exclusión, la muestra quedó conformada por 23 pacientes.

Todos fueron evaluados inicialmente con radiografías simples de frente y de perfil de la pierna, así como con una tomografía computarizada (TC) de la articulación de la rodilla o tobillo, según el extremo de la tibia comprometido (Figura 1). Tres casos eran fracturas expuestas, que fueron tratadas de urgencia, con una limpieza quirúrgica y estabilización inicial con fijador externo. Clasificamos las fracturas expuestas según Gustilo-Anderson. ${ }^{9}$ Los datos demográficos y las características de las fracturas se detallan en la Tabla.
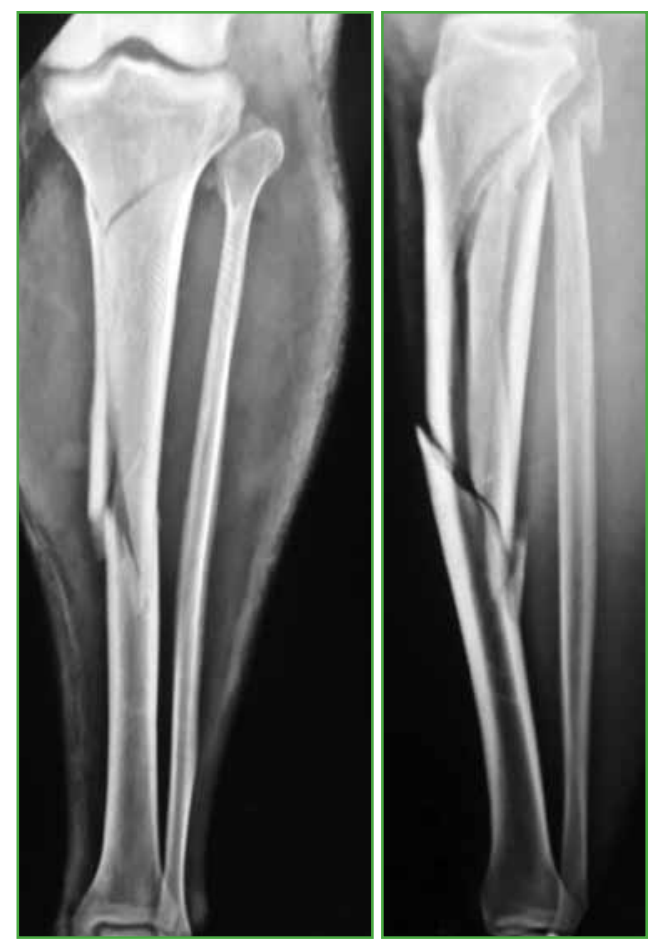

Figura 1. Caso 11. Fractura de tibia 41A.3 
Tabla. Datos demográficos

\begin{tabular}{|c|c|c|c|c|c|}
\hline & Sexo & Edad & Lado & Clasificación OTA & ¿Expuesta? \\
\hline 1 & M & 33 & I & 41A.2 & - \\
\hline 2 & M & 47 & D & $41 \mathrm{~A} .2$ & - \\
\hline 3 & $\mathrm{~F}$ & 29 & D & 43A. 1 & - \\
\hline 4 & M & 36 & I & $42 \mathrm{C} .2$ & 3B \\
\hline 5 & M & 50 & I & 43A. 3 & - \\
\hline 6 & M & 76 & I & 43A. 2 & - \\
\hline 7 & $\mathrm{~F}$ & 46 & D & 43A. 1 & - \\
\hline 8 & $\mathrm{~F}$ & 55 & I & $42 \mathrm{C} .2$ & 2 \\
\hline 9 & M & 26 & D & 43A.3 & - \\
\hline 10 & M & 48 & D & $41 \mathrm{~A} .2$ & - \\
\hline 11 & M & 35 & I & $41 \mathrm{~A} .3$ & - \\
\hline 12 & $\mathrm{~F}$ & 30 & D & 43A.2 & - \\
\hline 13 & M & 34 & D & 43A. 3 & - \\
\hline 14 & $\mathrm{~F}$ & 36 & I & 43A. 2 & - \\
\hline 15 & M & 50 & I & 41A. 3 & - \\
\hline 16 & $\mathrm{~F}$ & 76 & I & 43A. 2 & - \\
\hline 17 & M & 46 & D & 43A.1 & $3 \mathrm{~A}$ \\
\hline 18 & M & 55 & I & 43A.1 & - \\
\hline 19 & $\mathrm{~F}$ & 26 & D & 41A.2 & - \\
\hline 20 & M & 48 & D & 41A.2 & - \\
\hline 21 & M & 35 & I & 41A.2 & - \\
\hline 22 & M & 30 & $\mathrm{D}$ & 43A. & - \\
\hline 23 & $\mathrm{~F}$ & 34 & $\mathrm{D}$ & 43A.3 & - \\
\hline
\end{tabular}

Se evaluaron el dolor de rodilla, el rango de movilidad de la rodilla, el eje posoperatorio de la tibia y la tasa de consolidación de la fractura. Se utilizó la escala de Tegner-Lysholm para evaluar el impacto del dolor posoperatorio en las actividades de la vida diaria. ${ }^{10}$

El eje posoperatorio de la fractura fue evaluado en las radiografías completas de pierna tanto en la incidencia de frente como de perfil, mediante un goniómetro. Se consideró mala alineación o deseje a un ángulo $>5^{\circ}$ en cualquier plano. Los criterios utilizados en nuestro centro para considerar la consolidación ósea de las fracturas de tibia son la evidencia radiográfica de puentes de callo óseo, al menos, en tres de las cuatro corticales, sumado a la capacidad de marchar con carga sobre el miembro sin dolor.

El seguimiento promedio fue de 22 meses (mínimo 12 y máximo 38).

\section{Técnica quirúrgica}

Se ubica al paciente en posición supina, en una mesa radiolúcida para fracturas. Se coloca un realce debajo de la pierna afectada desde la rodilla hasta el tobillo, generando una flexión de $30^{\circ}$ de rodilla y de cadera. 
En los primeros casos de la serie, se realizó una incisión cutánea que se extendía unos 5 o $6 \mathrm{~cm}$, en forma oblicua, desde el borde interno del extremo distal del tendón rotuliano recorriendo el borde interno de la rótula. En los últimos casos de la serie, la incisión fue sobre la línea media, ligeramente medial. La incisión profunda se efectúa medial al tendón rotuliano y se extiende a través de los dos tercios distales del retináculo rotuliano medial. Esto permite la lateralización de la rótula y la exposición del punto de entrada del clavo (Figura 2).

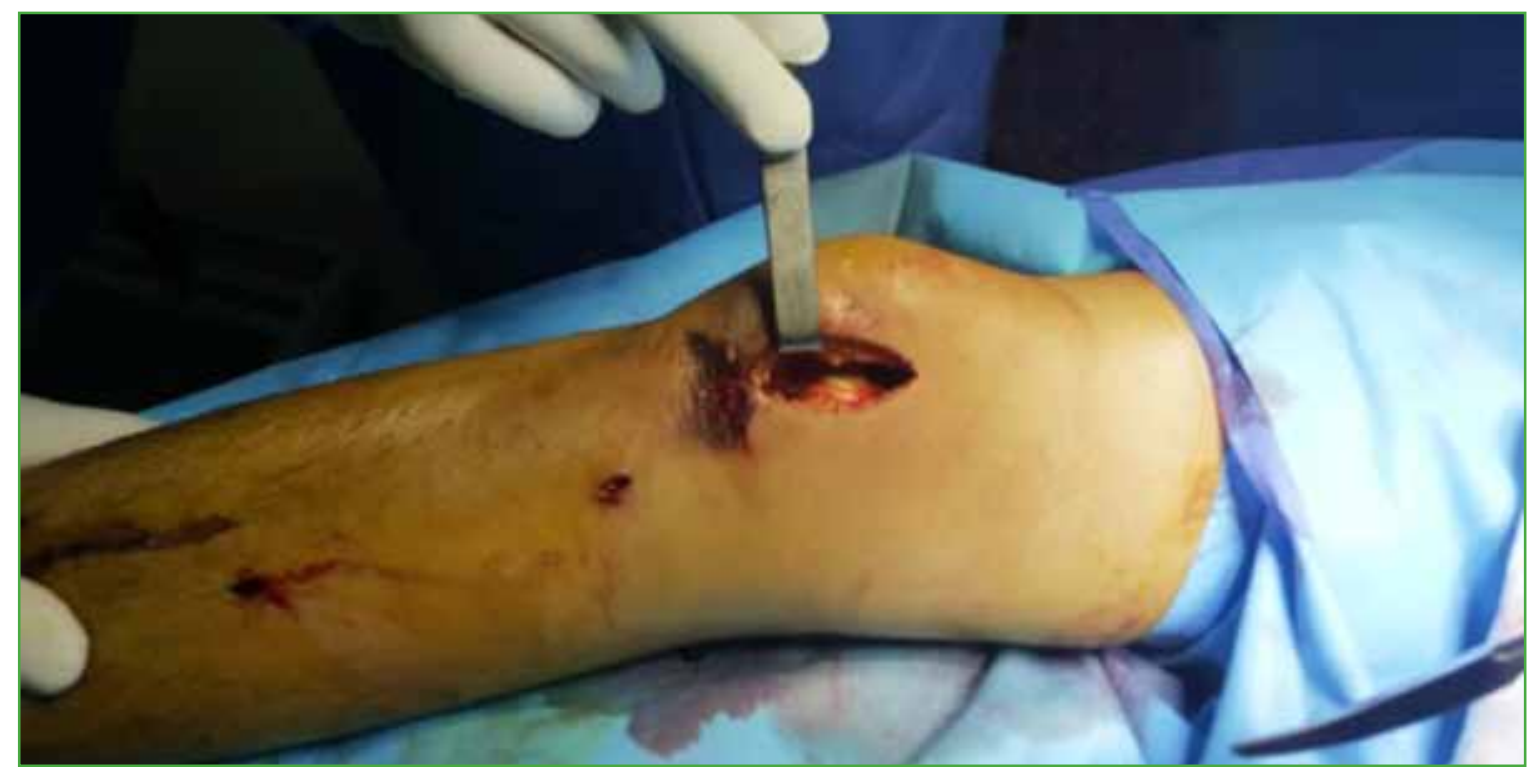

Figura 2. Abordaje pararrotuliano medial. Incisión en la piel ligeramente medial a la línea media y capsulotomía pararrotuliana interna.

Se emplearon diferentes estrategias (clamps percutáneos, tornillos poste, placas bloqueadas monocorticales, distractor femoral), según el patrón de la fractura, para lograr la reducción. La posición de la pierna en semiextensión también facilitó la reducción al neutralizar las fuerzas musculares deformantes. Para proteger el cartílago articular durante el fresado, se usó una jeringa de 20 mm (Figura 3).

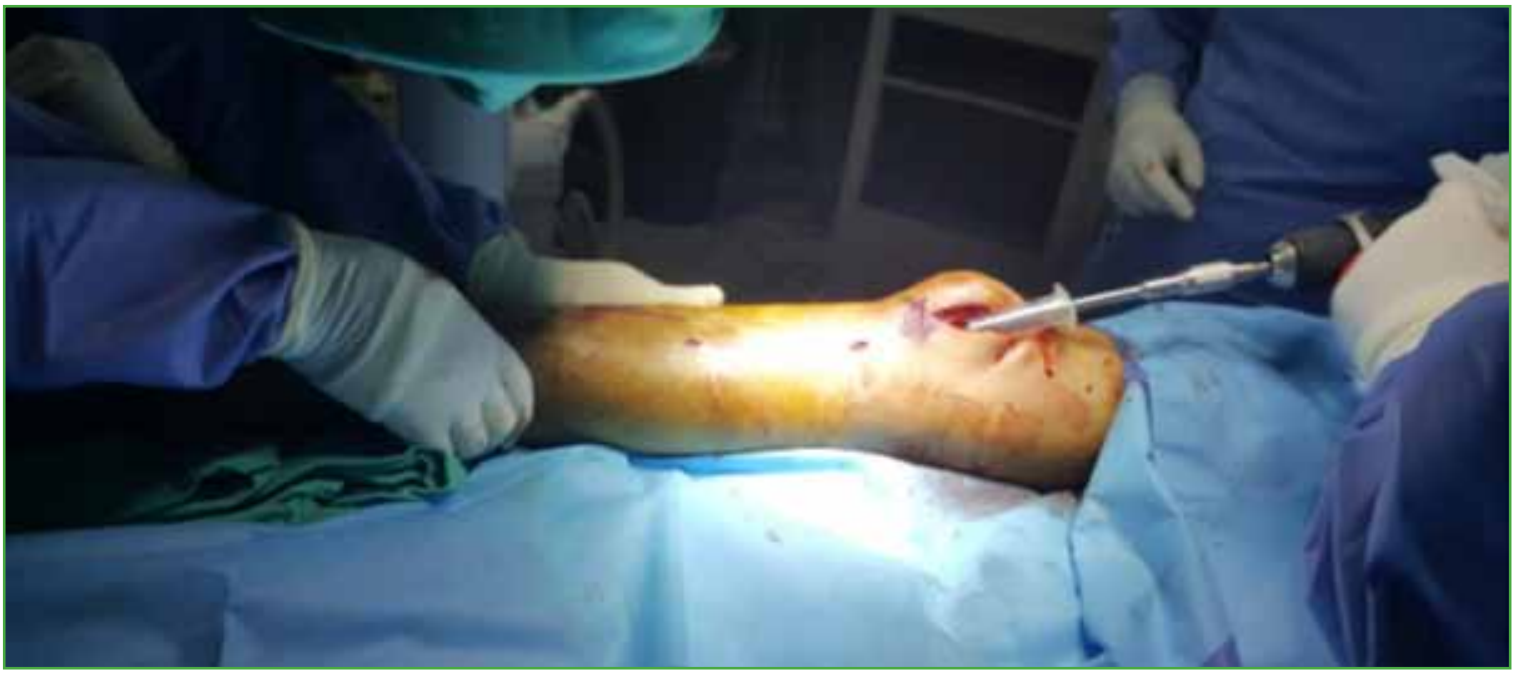

Figura 3. Fresado del canal en posición de semiextensión. 
El clavo se introduce con la pierna en semiextensión, evitando extender la rodilla para no dañar el cartílago articular (Figura 4). La cantidad y la configuración de los bloqueos se determinaron según las características de la fractura procurando, al menos, dos tornillos en el fragmento corto.

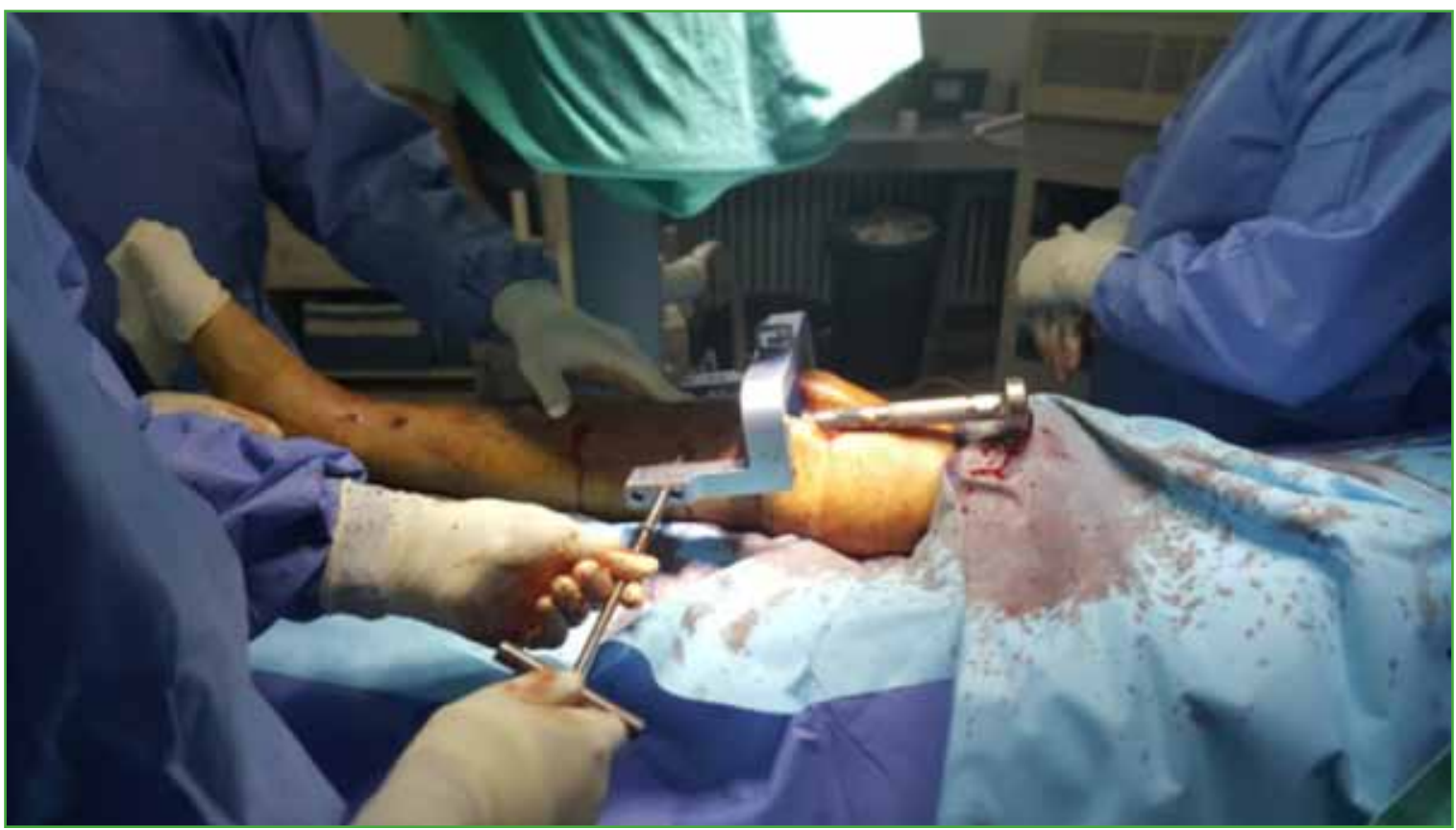

Figura 4. Introducción del clavo de tibia sin necesidad de modificar la posición de la pierna.

\section{Protocolo posoperatorio}

Todos los pacientes comenzaron precozmente con ejercicios y movilidad de la rodilla y el tobillo. Se indicó carga progresiva, según la tolerancia, con el uso de muletas.

\section{RESULTADOS}

\section{Movilidad articular}

$\mathrm{El}$ arco de movilidad de la rodilla, en el último control posoperatorio, fue de $125^{\circ}$ (rango 110-140) para la extremidad afectada en comparación con $135^{\circ}$ para la rodilla contralateral.

\section{Dolor anterior de rodilla}

Cuando se evaluó puntualmente el dolor de rodilla con la escala de Lysholm, 16 de los 23 pacientes no tenían dolor (25/25); cinco de 23 refirieron un leve dolor durante la actividad física (20/25) y los dos restantes obtuvieron puntajes $\leq 15$ sobre 25 .

\section{Deseje}

Se observó un eje posoperatorio inmediato adecuado $\left(<5^{\circ}\right)$ tanto en el frente como en el perfil, en todos los pacientes. Ninguno presentó un desplazamiento secundario en los subsiguientes controles.

\section{Consolidación}

Se logró la consolidación ósea en todos los casos, en un tiempo promedio de 5 meses (mínimo 3, máximo 10). Un paciente (4\%) con una fractura segmentaria (caso 4) requirió un aumento del foco proximal con una placa sobre el clavo más el aporte de injerto óseo autólogo para lograr la consolidación ósea (Figura 5). 

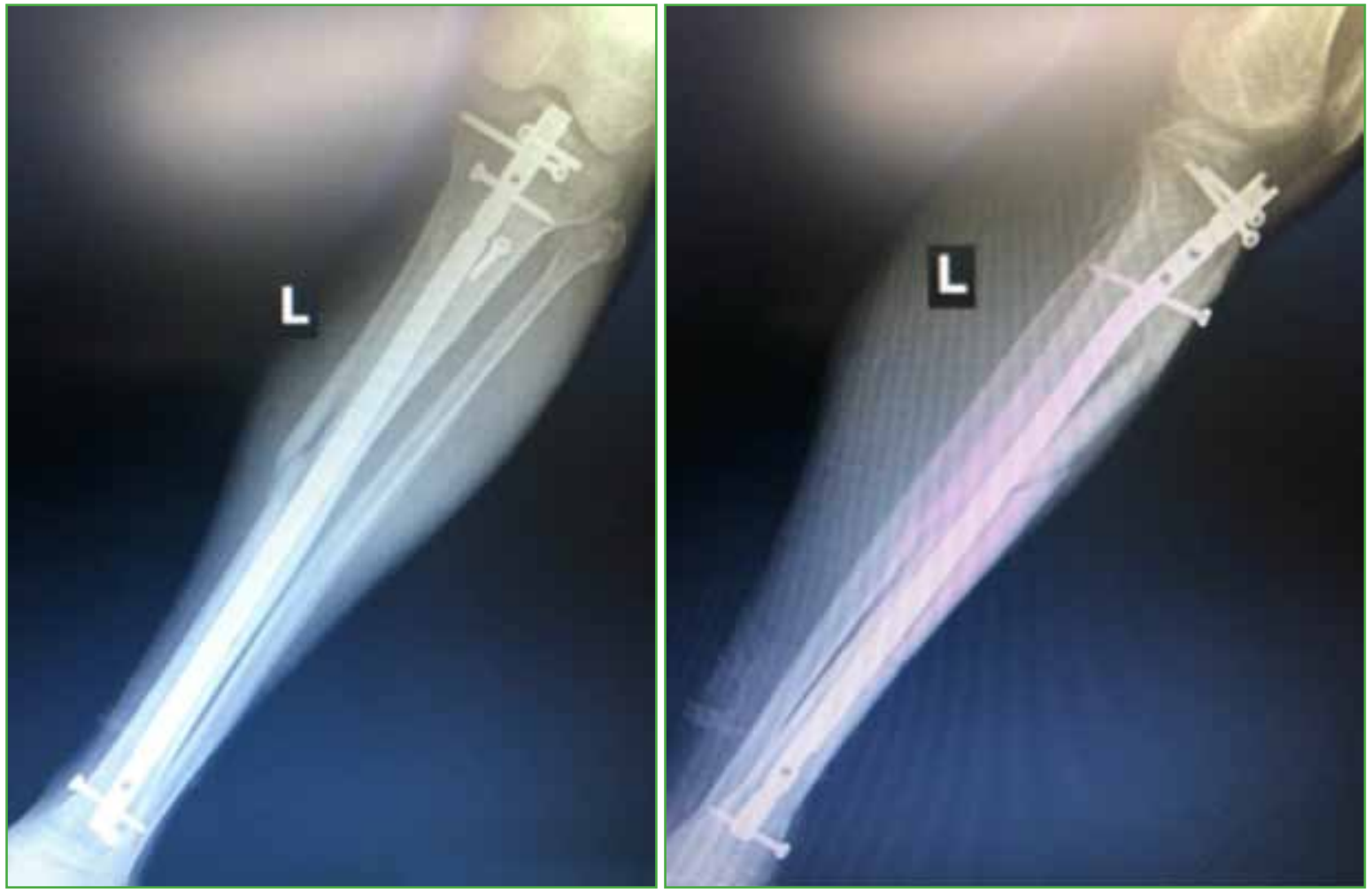

Figura 5. Imágenes del posoperatorio alejado. Se observan signos de consolidación con un correcto eje.

\section{DISCUSIÓN}

El enclavado endomedular es el tratamiento de elección para las fracturas diafisarias de tibia. Sin embargo, en las fracturas metafisarias, la falta de contacto cortical con el clavo en el ancho canal metafisario hace difícil lograr un buen resultado. Aunque hay reportes de desejes hasta del 58\% en las fracturas de tibia proximal tratadas con cla$\mathrm{vo}^{2,3,5} \mathrm{y}$ hasta del $29 \%$ en las de tibia distal, ${ }^{11}$ el desarrollo de nuevas técnicas quirúrgicas y las mejoras en el diseño de los clavos han equiparado los resultados con el de las placas. En la actualidad, no hay estudios comparativos que hayan demostrado alguna diferencia clara en los resultados a favor del tratamiento con placas o clavos en estas fracturas. Sin embargo, debido a las ventajas biomecánicas de los clavos sobre las placas, elegimos el enclavado endomedular como nuestra primera opción terapéutica para las fracturas proximales o distales de tibia.

En 2006, Nork y cols. publicaron los resultados de una serie de 37 pacientes con fracturas proximales de tibia tratadas con clavo endomedular mediante la técnica infrarrotuliana. Resaltan la dificultad de mantener la reducción durante el procedimiento y que, en 27 de los 37 casos, fue necesario utilizar métodos adicionales para asistir en la reducción, como una placa monocortical o un distractor femoral. En 34 de los 37 casos, lograron un correcto eje posoperatorio. ${ }^{12}$

Con la intención de simplificar la técnica se han desarrollado abordajes alternativos, como el suprarrotuliano ${ }^{8} \mathrm{o}$ pararrotuliano, ${ }^{6}$ para la introducción del clavo en una posición de semiextensión de rodilla, de manera de neutralizar la acción del aparato extensor y permitir la colocación del clavo sin la necesidad de manipular la pierna durante el fresado y la introducción del implante.

En 1996, Tornetta y cols., ${ }^{6}$ desarrollaron el abordaje pararrotuliano medial, el cual permite lateralizar la rótula e introducir el clavo en posición de semiextensión y así disminuir la posibilidad de dañar el cartílago articular rotulofemoral. Consiguieron un correcto eje en 23 de los 25 pacientes de la serie. Como ventaja adicional mencionan menos tiempo de uso de la radioscopia al compararla con la técnica infrarrotuliana clásica.

Otro de los abordajes más modernos descritos para colocar un clavo de tibia en semiextensión es el suprarrotuliano, a través de una incisión en el tendón cuadricipital. ${ }^{8}$ Este abordaje requiere el uso de un set de colocación específico para evitar el daño del cartílago de la articulación rotulofemoral, durante el fresado y la introducción 
del clavo. En 2014, Sanders y cols. prácticamente no reportaron daños en la articulación rotulofemoral luego del enclavado endomedular de 51 fracturas diafisarias de tibia a través de un portal suprarrotuliano. Destacan que la tasa de dolor anterior de rodilla posoperatorio en su serie fue incluso menor que las reportadas con la técnica tradicional infrarrotuliana. ${ }^{13}$

Ese mismo año, Tornetta y cols. publicaron una serie de 185 fracturas de tibia, 84 fracturas metafisarias de tibia proximal y distal tratadas con enclavado endomedular a través de un abordaje pararrotuliano medial. En todos los casos, comunicaron un correcto eje posoperatorio. Así mismo, evaluaron el dolor posoperatorio de rodilla y no hallaron diferencias significativas a los 2.3 años con respecto a la técnica infrarrotuliana. ${ }^{14}$

En 2019, Kubiak y cols. presentaron un estudio comparativo aleatorizado sobre el dolor de rodilla posoperatorio con la técnica pararrotuliana lateral en semiextensión y con la técnica infrarrotuliana clásica. Los resultados revelaron menos dolor de rodilla en el grupo de pacientes operados con la técnica pararrotuliana. ${ }^{15}$

Ibrahim y cols. publicaron una revisión bibliográfica, en la que incluyeron principalmente estudios comparativos entre la técnica en semiextensión y la técnica infrarrotuliana tradicional, y concluyeron en que los resultados de dolor posoperatorio y función de rodilla son mejores con la técnica en semiextensión. ${ }^{16}$

Finalmente, debido a la preocupación que podría generar un abordaje intrarticular, incluso en las fracturas expuestas de tibia, Mitchel y cols. estudiaron 131 fracturas expuestas de tibia tratadas con un clavo endomedular de tibia a través de un abordaje suprarrotuliano y comunicaron un aumento del riesgo de artritis séptica en comparación con aquel publicado con la técnica infrarrotuliana. ${ }^{17}$

$\mathrm{Al}$ igual que los otros autores, hemos encontrado más simple lograr la reducción de la fractura y mantenerla, durante el fresado del canal y la introducción del clavo. Asimismo nos permitió, cuando fue necesario, realizar, en primera instancia, el acerrojado distal del clavo sin modificar la posición de la pierna para efectuar la compresión de la fractura con la técnica de backslap. También, destacamos como una ventaja del abordaje, la posibilidad de hacer todo el procedimiento con el set de colocación habitual, esto no es posible con la técnica suprarrotuliana. Por último, creemos que otra ventaja de esta técnica es la posibilidad de realizar un uso del arco en C más preciso y eficiente.

Como limitaciones de nuestro estudio, podemos mencionar la falta de una evaluación más sistemática y profunda de la articulación rotulofemoral posoperatoria, el bajo número de la serie y la falta de un grupo control con la técnica tradicional infrarrotuliana.

\section{CONCLUSIÓN}

La colocación de un clavo para el tratamiento de las fracturas metafisarias de tibia a través de un abordaje pararrotuliano medial con la rodilla en semiextensión, resulta en una técnica segura y simple que permite conseguir excelentes resultados de consolidación y eje posoperatorio, sin aumentar el dolor de rodilla posoperatorio.

Conflicto de intereses: Los autores no declaran conflictos de intereses.

\section{BIBLIOGRAFÍA}

1. Bone LB, Johnson KD. Treatment of tibial fractures by reaming and intramedullary nailing. J Bone Joint Surg Am 1986;68:877-87. PMID: 3733776

2. Freedman EL, Johnson EE. Radiographic analysis of tibial fracture malalignment following intramedullary nailing. Clin Orthop Relat Res 1995;(315):25-33. PMID: 7634677

3. Lang GJ, Cohen BE, Bosse MJ, Kellam JF. Proximal third tibial shaft fractures: should they be nailed? Clin Orthop Relat Res 1995;(315):64-74. PMID: 7634688 
4. Lindvall E, Sanders R, DiPasquale T, Herscovici D, Haidukewych G, Sagi C. Intramedullary nailing versus percutaneous locked plating of extra-articular proximal tibial fractures: comparison of 56 cases. J Orthop Trauma 2009;23(7):485-92. https://doi.org/10.1097/BOT.0b013e3181b013d2

5. Yaligod V, Rudrappa GH, Nagendra S, Shivanna UM. Minimizing the complications of intramedullary nailing for distal third tibial shaft and metaphyseal fractures. J Orthop 2013;11(1):10-8. https://doi.org/10.1016/j.jor.2013.12.002

6. Tornetta P $3^{\text {rd }}$, Collins E. Semiextended position of intramedullary nailing of the proximal tibia. Clin Orthop 1996;(328):185-9. https://doi.org/10.1097/00003086-199607000-00029

7. Kubiak EN, Widmer BJ, Horwitz DS. Extra-articular technique for semiextended tibial nailing. J Orthop Trauma 2010;24(11):704-8. https://doi.org/10.1097/BOT.0b013e3181d5d9f4

8. Cole JD. Distal tibia fracture: Opinion: intramedullary nailing. J Orthop Trauma 2006;20:73-4. https://doi.org/10.1097/01.bot.0000196658.55293.e6

9. Gustilo RD, Anderson JT. Prevention of infection in the treatment of one thousand and twenty-five open fractures of long bones: retrospective and prospective analysis. J Bone Joint Surg Am 1976;58:453-8. PMID: 773941

10. Collins NJ, Misra D, Felson DT, Crossley KM, Roos EM. Measures of knee function: International Knee Documentation Committee (IKDC) Subjective Knee Evaluation Form, Knee Injury and Osteoarthritis Outcome Score (KOOS), Knee Injury and Osteoarthritis Outcome Score Physical Function Short Form (KOOS-PS), Knee Outcome Survey Activities of Daily Living Scale (KOS-ADL), Lysholm Knee Scoring Scale, Oxford Knee Score (OKS), Western Ontario and McMaster Universities Osteoarthritis Index (WOMAC), Activity Rating Scale (ARS), and Tegner Activity Score (TAS). Arthritis Care Res (Hoboken) 2011;63(Suppl 11):S208-28. https://doi.org/10.1002/acr.20632

11. Vallier HA, Le TT, Bedi A. Radiographic and clinical comparison of distal tibia shaft fractures (4 to $11 \mathrm{~cm}$ proximal to the plafond): plating versus intramedullary nailing. J Orthop Trauma 2008;22(5):307-11. https://doi.org/10.1097/BOT.0b013e31816ed974

12. Nork SE, Barei DP, Schildhauer TA. Intramedullary nailing of proximal quarter tibial fractures. J Orthop Trauma 2006;20(8):523-8. https://doi.org/10.1097/01.bot.0000244993.60374.d6

13. Sanders RW, DiPasquale TG, Jordan CJ, Arrington JA, Sagi HC. Semiextended intramedullary nailing of the tibia using a suprapatellar approach: radiographic results and clinical outcomes at a minimum of 12 months follow-up. J Orthop Trauma 2014;28(5):245-55. https://doi.org/10.1097/BOT.0000000000000082

14. Ryan SP, Steen B, Tornetta P $3^{\text {rd }}$. Semi-extended nailing of metaphyseal tibia fractures: alignment and incidence of postoperative knee pain. J Orthop Trauma 2014;28(5):263-9. https://doi.org/10.1097/BOT.0000000000000083

15. Rothberg DL, Stuart AR, Presson AP, Haller JM, Higgins TF, Kubiak EN. A comparison of the open semi-extended parapatellar versus standard entry tibial nailing techniques and knee pain: a randomized controlled trial. J Orthop Trauma 2019;33(1):31-6. https://doi.org/10.1097/BOT.0000000000001309

16. Ibrahim I, Johnson A, Rodriguez EK. Improved outcomes with semi-extended nailing of tibial fractures? A systematic review. J Orthop Trauma 2019;33(3):155-60. https://doi.org/10.1097/BOT.0000000000001395

17. Mitchell PM, Weisenthal BM, Collinge CA. No incidence of postoperative knee sepsis with suprapatellar nailing of open tibia fractures. J Orthop Trauma 2017;31(2):85-9. https://doi.org/10.1097/BOT.0000000000000725 